Metal-Organic Frameworks: Literature Survey and Recommendation of Potential Sorbent Materials

T. F. Baumann

May 3, 2010 
This document was prepared as an account of work sponsored by an agency of the United States government. Neither the United States government nor Lawrence Livermore National Security, LLC, nor any of their employees makes any warranty, expressed or implied, or assumes any legal liability or responsibility for the accuracy, completeness, or usefulness of any information, apparatus, product, or process disclosed, or represents that its use would not infringe privately owned rights. Reference herein to any specific commercial product, process, or service by trade name, trademark, manufacturer, or otherwise does not necessarily constitute or imply its endorsement, recommendation, or favoring by the United States government or Lawrence Livermore National Security, LLC. The views and opinions of authors expressed herein do not necessarily state or reflect those of the United States government or Lawrence Livermore National Security, LLC, and shall not be used for advertising or product endorsement purposes.

This work performed under the auspices of the U.S. Department of Energy by Lawrence Livermore National Laboratory under Contract DE-AC52-07NA27344. 


\section{Metal-Organic Frameworks: Literature Survey and Recommendation of Potential Sorbent Materials}

Theodore F. Baumann

Lawrence Livermore National Laboratory

\section{Executive Summary}

Metal-organic frameworks (MOFs) are a special type of porous material with a number of unique properties, including exceptionally high surface areas, large internal pore volumes (void space) and tunable pore sizes. These materials are prepared through the assembly of molecular building blocks into ordered three-dimensional structures. The bulk properties of the MOF are determined by the nature of the building blocks and, as such, these materials can be designed with special characteristics that cannot be realized in other sorbent materials, like activated carbons. For example, MOFs can be constructed with binding sites or pockets that can exhibit selectivity for specific analytes. Alternatively, the framework can be engineered to undergo reversible dimensional changes (or "breathing") upon interaction with an analyte, effectively trapping the molecule of interest in the lattice structure. In this report, we have surveyed the 4000 different MOF structures reported in the open literature and provided recommendations for specific MOF materials that should be investigated as sorbents for this project.

\section{Overview}

Metal-organic frameworks (MOFs) are a unique class of crystalline porous solids that have been studied extensively for a number of applications, including gas storage, gas separation and heterogeneous catalysis. ${ }^{1}$ Materials of this type are prepared using an approach known as "reticular chemistry" which involves linking of molecular building blocks into predetermined architectures that are held together by strong bonds. In MOF structures, organic linkers serve as rigid "struts" that bridge inorganic "joints" to form extended three-dimensional networks (Figure 1). Unlike other porous materials, MOFs are open scaffolds that have pores without walls, a feature that endows these materials with exceptionally large surface areas. In addition, since the topology of the scaffold is defined by molecular building blocks, the pore or aperture size within the framework is quite small, typically less than $20 \AA$, leading to strong interactions between guest 
molecules and the framework. The porosity of MOFs is greater than that of any other porous material, including activated carbons, making MOFs ideal candidates for use as adsorbents. The objective of this report is to survey the vast number of MOF structures reported in the open literature to select candidate materials for use as sorbents in this project. The reticular chemistry approach has been used with a wide variety of struts and joints, leading to over 4,000 reported MOF structures as of

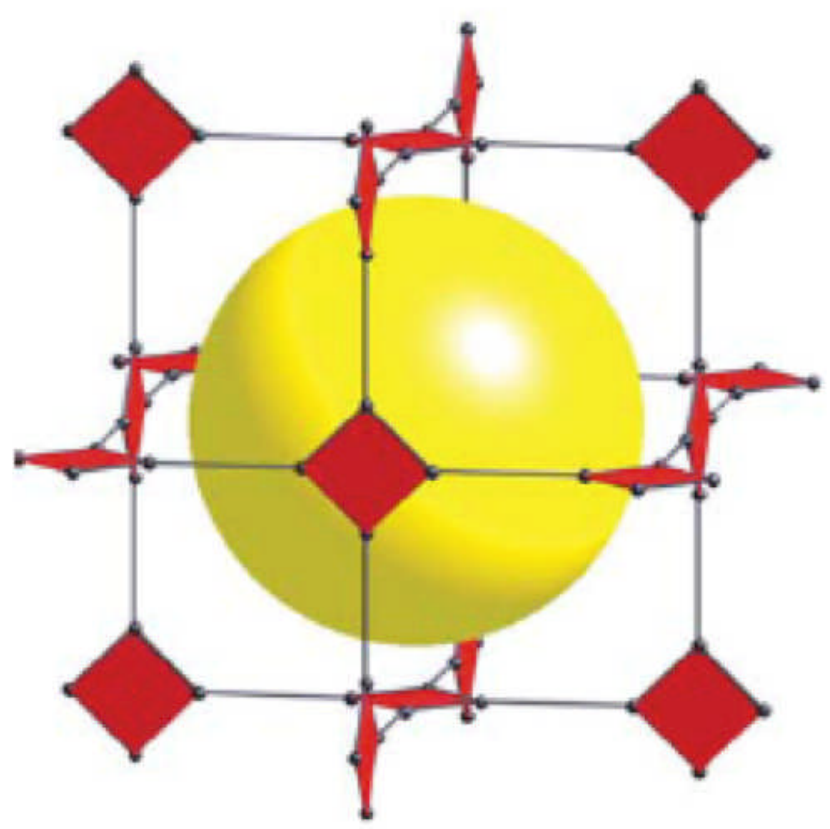

Figure 1. Schematic representation of a MOF unit cell showing the arrangement of inorganic joints (red squares) linked by organic struts (black rods). The yellow sphere represents the void space within the lattice framework (ref. 4). 2009. ${ }^{2}$ This large set of materials were reviewed using the following criteria relevant to the desired application:

- Surface Area: MOFs with higher surface area are more desirable

- Pore Size: MOFs must have the proper pore size to allow uptake and release of analytes

- Stability: MOFs must exhibit reasonable stability upon exposure to oxygen, moisture, the analytes of interest or changes in temperature

- Solubility: MOFs should be insoluble in aqueous media

- Analyte Interaction: MOFs may exhibit special structural characteristics that may facilitate selective uptake and release of analytes

In this report, we describe the processes used to synthesize MOF structures, the different classes of MOF materials and their physical properties, and make recommendations of MOF systems that warrant further evaluation for this project. 


\section{MOF Chemistry}

MOFs are prepared as crystalline solids by solution reactions of metal salts with organic linkers. Typically, the organic linkers are capable of connecting two metal oxide clusters (ditopic linkers), such as benzenedicarboxylate (BDC), but linkers with higher dimensionality can also be used (Figure 2). The bonds formed between the metal ions and the donor atoms of the linker are strong and, as a result, the extended network structure in the MOF is quite robust. The coordination complex formed by the metal

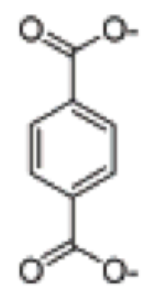

$\mathrm{BDC}$

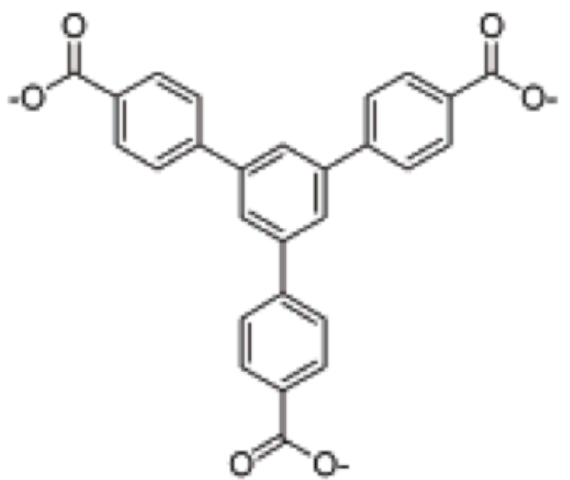

BTB

Figure 2. Examples of ditopic (BDC: 1,4benzenedicarboxylate) and tritopic (BTB: 1,3,5benzenetribenzoate) organic linker used in the construction of MOFs (ref 3a). ions and the donor atoms of the linker, also termed the secondary building unit (SBU), dictates the final topology of the MOF framework. For example, copper-carboxylate clusters, in which two copper atoms are chelated by four carboxylates $\left[\mathrm{Cu}_{2}\left(\mathrm{O}_{2} \mathrm{C}-\right)_{4}\right]$, can be considered square SBUs as the four carbon atoms of the carboxylate linkers form the points of a square that can then be used as tetra-functional joint in the construction of various network structures (Figure 3). Conversely, zinc-carboxylate clusters $\left[\mathrm{Zn}_{4} \mathrm{O}\left(\mathrm{O}_{2} \mathrm{C}-\right)_{6}\right]$ are octahedral SBUs that can be used as six-coordinate joints. The stability and rigidity of the SBUs allows for their reticulation with the appropriate linker into MOF architectures with predetermined structural motifs. The octahedral zinc-carboxylate SBU, when combined with the linear 1,4-BDC linker, yields an extended cubic structure, known as MOF-5 (Figure 4). ${ }^{3}$ In the unit cell for this material, eight $\mathrm{Zn}_{4} \mathrm{O}$ SBUs are bridged by twelve BDC linkers to define a large internal cavity. Due to this open structure, MOF-5 has a surface area of 3,800 $\mathrm{m}^{2} / \mathrm{g}$ and $60 \%$ of the crystal is void space that can be used to hold guest molecules. 


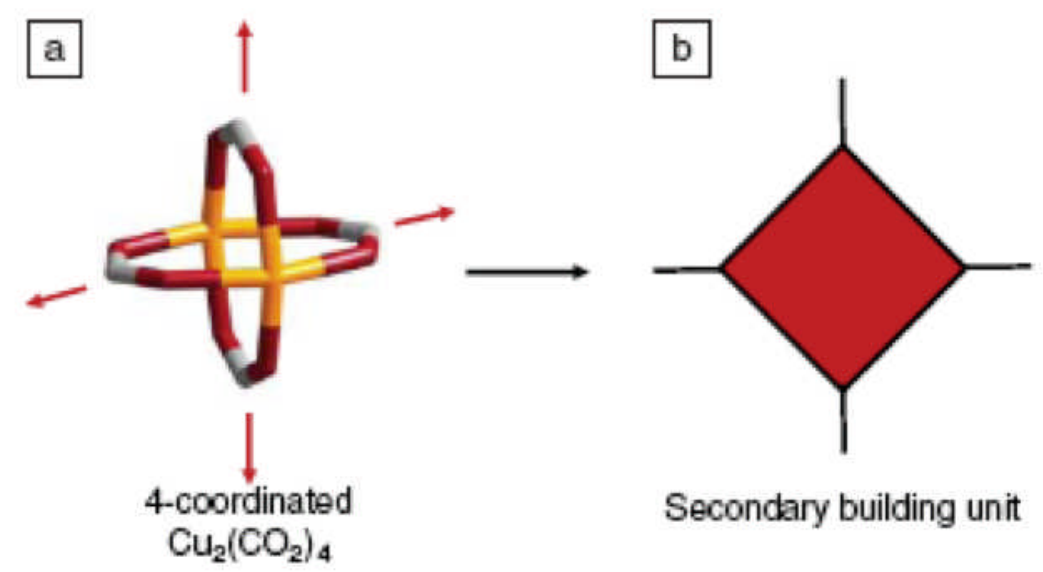

Figure 3. Structural representations of a $\mathrm{Cu}_{2}\left(\mathrm{O}_{2} \mathrm{C}-\right)_{4}$ secondary building unit (SBU), in which the copper atoms (yellow) are coordinated by four carboxylate units (oxygen in red and carbon in grey), creating a tetra-functional square SBU (ref 4).

One of the powerful aspects of reticular chemistry is that a wide variety of MOF crystal structures can be accessed by changing the size and dimensionality of the organic linker or the coordination geometry of the SBU. In the case of MOF-5, a series of isoreticular MOFs (i.e. MOFs with the same cubic symmetry but different pore sizes) can be readily prepared simply by changing the length of the organic spacer. ${ }^{4}$ Interestingly, MOF systems prepared with longer organic linkers will often form interpenetrating frameworks to maximize packing efficiency in the crystal. Interpenetration of lattices leads to smaller pore sizes in the MOF structure, but also decreases the total pore volume for the material. Changing the dimensionality of the linker can be used to increase the surface area of MOF structures. For example, combining a tritopic linker $(1,3,5-$ benzenetricaboxylate, BTC) with the same Zn SBU as used in MOF-5 generates a material with a surface area of 5,500 $\mathrm{m}^{2} / \mathrm{g}$, known as MOF-177. ${ }^{5}$ Different crystalline frameworks can also be synthesized by changing the geometry of the SBU. Beyond the square and octahedral SBUs described above, a variety of other SBU shapes, including trigonal, tetrahedral and trigonal bipyramid, can be prepared depending on the metal ion and the donor atoms. These different SBU shapes, when combined with the appropriate linker, allows for the tailored design of MOFs with crystalline networks that range from layered graphitic structures to 3-D hexagonal cages. 
To utilize these materials as sorbents, the MOF structure must first be "activated". This process involves removal of solvent molecules from the pores of the MOF to open the void space in the crystal lattice. Typically, the majority of included solvent can be removed from the structure through evaporation. More rigorous techniques, however, are often required to evacuate solvent or guest molecules that have stronger interactions with the organic struts or inorganic clusters. Therefore, most MOFs have to be activated under vacuum at elevated temperatures $\left(>200^{\circ} \mathrm{C}\right)$ to eliminate the strongly bound solvent molecules. The fact that these materials can be dried at these temperatures underscores the robust nature of these structures. Most MOF materials are thermally stable to temperatures as high as $400^{\circ} \mathrm{C}$, with some special types of MOFs exhibiting stability over $500^{\circ} \mathrm{C}$. In addition, these materials are quite stable to a variety of chemical environments. MOFs are
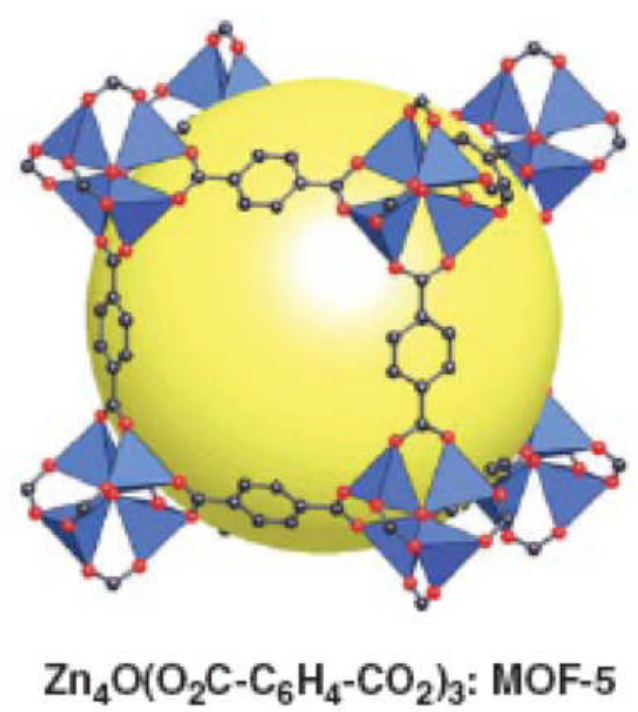

Figure 4. The unit cell for the MOF-5 framework in which the $\mathrm{Zn}$ clusters are shown in blue, the oxygen atoms in red and the linker units in black (ref 4).

insoluble in water, most organic solvents and can even retain their crystalline structure after treatment in strongly basic solutions. In certain MOFs, the organic linker of the framework can be modified through solution-based techniques without destruction of the crystal lattice. ${ }^{6}$ In fact, MOFs have even been used as catalysis for both gas- and liquidphase chemical transformation. ${ }^{7}$ Many MOF structures, however, are digested when treated with mild acid, especially those constructed from carboxylate SBUs. This digestion process may be useful in the recovery of guest analytes that adsorb strongly to the MOF framework.

\section{Recommendations}

As stated previously, over 4,000 different MOF structures have been reported in the literature. For the purposes of this report, we surveyed the literature and, based on the set of criteria described above, identified three classes of MOF materials that are viable 
candidates for this project: (1) traditional metal-carboxylate MOFs, (2) zeoliticimidazolate frameworks (ZIFs), and (3) covalent-organic frameworks (COFs). In the following sections, we provide details regarding each class of MOF and make recommendation for specific MOFs that should be investigated as part of this effort. The candidate systems, along with their physical properties, are listed in Table 1. While a few MOF structures are available from commercial suppliers (4 MOF products manufactured by BASF and distributed by Aldrich), we believe the characteristics of these commercially available materials are not well suited for this particular project.

Table 1. Physical properties for the candidate MOF materials

\begin{tabular}{lcccl} 
MOF & SA $\left(\mathrm{m}^{2} / \mathrm{g}\right)$ & Pore size $(\AA)$ & $\mathbf{T}_{\mathbf{s}}\left({ }^{\circ} \mathrm{C}\right)$ & Special Characteristics \\
\hline MOF-5 & 3,800 & 10 & 400 & High surface area \\
MOF-177 & 5,500 & 11 & 400 & High surface area \\
MIL-101 & 5,900 & 30 & 400 & High surface area \\
HKUST-1 & 2,100 & 9 & 350 & Open Cu coordination sites \\
MOF-74 & 820 & 11 & 400 & Open Zn coordination sites \\
ZIF-8 & 2,000 & 11 & 550 & Small pore apertures $(3.4 \AA)$ \\
COF-103 & 4,200 & 12 & 500 & High surface area \\
\hline
\end{tabular}

For the traditional metal-carboxylate MOF materials, we focused on a small subset of materials that exhibit exceptionally high surface areas. As described earlier, the Zncarboxylate systems, MOF-5 and MOF-177, have surface areas of 3,800 and 5,500 $\mathrm{m}^{2} / \mathrm{g}$ respectively, and, as such, should be tested as sorbents. MOF-5 has a cubic network structure with square pores that are $10 \AA$ in diameter, while MOF-177 possesses a more complex 3-D cage structure with a pore diameter of $11 \AA$. In addition to those two materials, MIL-101, a Cr-carboxylate system with a surface area of 5,900 $\mathrm{m}^{2} / \mathrm{g}$, also appears to be a promising candidate for this project. $^{8}$ This material has a cubic cage 
structure in which $\left[\mathrm{Cr}_{3} \mathrm{~F}\left(\mathrm{H}_{2} \mathrm{O}\right)_{2} \mathrm{O}\right]$ inorganic joints are linked by 1,4-DBC struts, creating two distinct cage morphologies (inner diameters of 29 and $34 \AA$ ) that are connected by smaller apertures (diameters of 12 and $16 \AA$ ). All three of these MOF materials have been shown to excellent sorbent for gases, such as hydrogen, methane and carbon dioxide. Within the metal-carboxylate MOFs family, a second type of material that may prove useful for this project are those that have been engineered to have metal clusters with open coordination sites. When activated, these open metal sites serve as Lewis acid sites for interaction with guest molecules and can therefore be exploited to increase the binding energy or selectivity of the MOF for specific analytes. Among the various MOFs that have been designed with open metal sites, we recommend two candidate materials be investigated as sorbents. HKUST-1 is a copper-carboxylate MOF with a surface area of $2100 \mathrm{~m}^{2} / \mathrm{g}$ and hexagonal network of $9 \AA$ pores. ${ }^{9}$ The copper atoms adjacent to the hexagonal channels contain axial water molecules that can be removed by heating the sample under vacuum, exposing the $\mathrm{Cu}$ sites in the MOF. Likewise, MOF-74 is a zinccarboxylate structure with a surface area of $800 \mathrm{~m}^{2} / \mathrm{g}$, hexagonal channels with a diameter of $11 \AA$, and open $\mathrm{Zn}$ sites that can be exposed upon dehydration. ${ }^{10}$ MOF-74 has been tested as a sorbent for harmful gases, such sulfur dioxide, chlorine, ammonia and benzene, and outperformed activated carbon both in terms of capacity and selectivity. ${ }^{11}$ The one caveat associated with this type of MOF is that the materials will have to be stored in an inert environment prior to use, as the open coordination sites will readily scavenge guest molecules (water, oxygen, etc) if exposed to ambient conditions. 
The second class of MOF materials recommended as sorbent candidates are zeolitic-imidazolate frameworks (ZIFs). ${ }^{12}$ These materials differ from metal-carboxylate MOFs in that the SBUs consist of metal ions coordinated by imidazolate linkers as opposed to carboxylates, leading to the formation crystalline tetrahedral networks similar
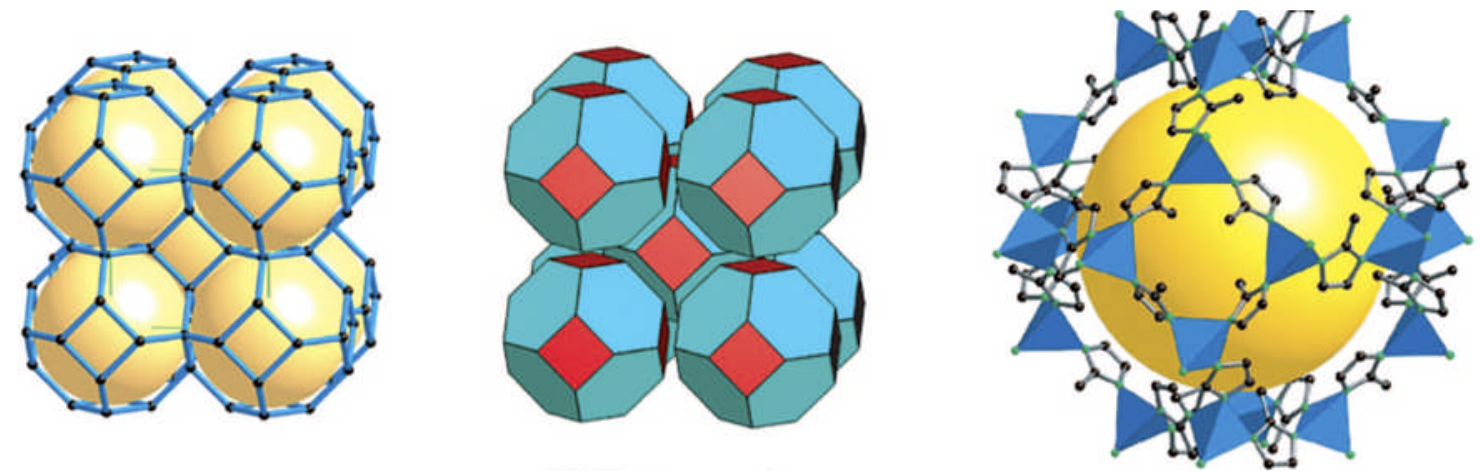

Figure 5. Different structural representations of ZIF-8 showing both the sodalite structure and the coordination geometry of the Zn-imidazolate complex (blue tetrahedra) (ref. 12b).

to those found in traditional inorganic zeolite structures. A wide variety of zeolitic structures can be accessed with different metals and imidazolate linkers using reticular chemistry. These materials exhibit higher thermal stability than traditional MOFs (up to $550^{\circ} \mathrm{C}$ ) and remarkable chemical resistance to organic solvents and even boiling alkaline water. The surface areas for ZIFs (up to $2000 \mathrm{~m}^{2} / \mathrm{g}$ ) are not as high as those reported for metal-carboxylate MOFs, but the coordination geometry associated with the ZIF building blocks defines smaller pore apertures in these structures, some as small as $3 \AA$. The ability to control the aperture diameter may be used to engineer size selectivity into the ZIF sorbents. Among the various ZIF materials reported, ZIF-8 appears to be a promising candidate for use as a sorbent material. ZIF-8 is a Zn-imidazolate complex with a surface are $\sim 2000 \mathrm{~m}^{2} / \mathrm{g}$ and a sodalite structure that defines cages that have a diameter of $11.6 \AA$ connected by $3.4 \AA$ apertures (Figure 5). ${ }^{12}$ Due to its large internal pore volume, this material has shown promise as a sorbent for hydrogen storage as well as carbon dioxide capture.

The final class of MOF materials that warrant investigation as part of this project are covalent-organic frameworks (COFs). ${ }^{13}$ Unlike traditional MOFs and ZIFs, COF materials do not rely on inorganic SBUs for the formation of extended networks. As the name implies, these materials are composed entirely of organic elements, typically carbon, boron and oxygen, covalently linked with one another by strong bonds into 
ordered porous frameworks. By choosing the right organic building blocks and linking reactions, crystalline solids that exhibit high surface areas, large pore volumes and high thermal stabilities can be prepared. The network structures in COFs are formed by condensation reactions of trigonal or tetrahedral boronic acid derivatives or cocondensation of boronic acids with aromatic diols. Dehydration that occurs during condensation leads to the formation of stable boroxine $\left(\mathrm{B}_{3} \mathrm{O}_{3}\right)$ rings or boronate ester $\left(\mathrm{C}_{2} \mathrm{O}_{2} \mathrm{~B}\right)$ rings that link together the rigid organic struts. For this project, we recommend that a representative COF (COF-103) be investigated as a sorbent material (Figure 6). COF-103 exhibits an exceptionally high surface area $\left(4200 \mathrm{~m}^{2} / \mathrm{g}\right)$ and extremely large internal pore volumes. ${ }^{13 \mathrm{~b}}$ This material has a 3-D cage structure that defines pores with diameters $\sim 12.5 \AA$. Like most other COFs, this material is thermally stable up to $500^{\circ} \mathrm{C}$ and insoluble in water and organic solvents. As with the MOFs and ZIFs, these materials have shown promise as sorbents for a variety of gases.

Based on the results for these seven candidate MOFs, future studies can focus on next-generation MOFs that have been modified to improve either the capacity or selectivity of

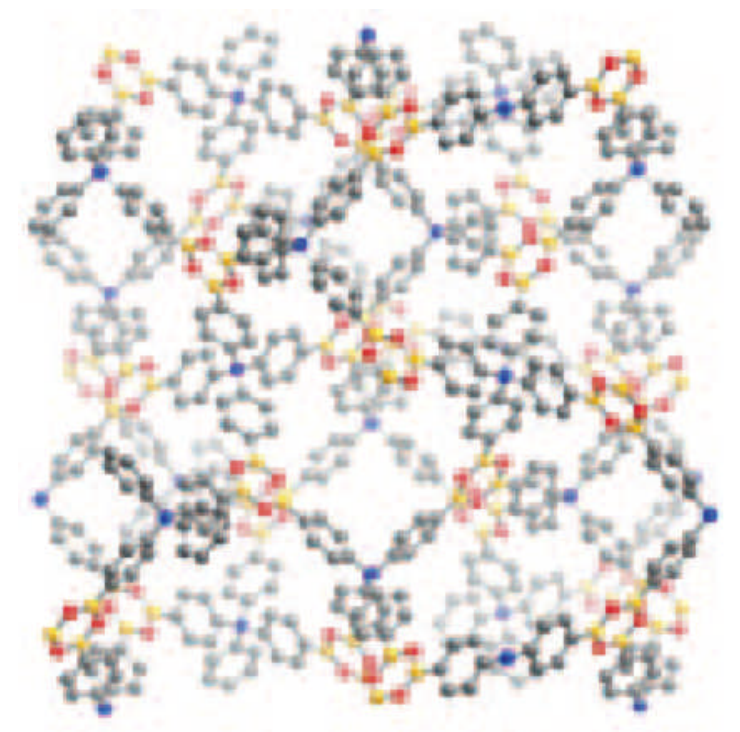

Figure 6. Crystal structure of COF-103 showing the organic building blocks interconnected by the boroxine rings ( $\mathrm{B}$ in yellow, $\mathrm{O}$ in orange, $\mathrm{C}$ in grey) (ref. 13c). the MOF for a particular analyte. For example, the chemical functionality of the organic struts in either the MOF or ZIF materials can be changed to increase the binding energy of an analyte to the framework (similar to the open metal sites in HKUST-1 or MOF-74). Alternatively, chemical functionality on the struts can be used to "gate" the pores of the MOF material, allowing for either selective binding of analytes or trapping of species in the pores. Along these same lines, MOF frameworks can be engineered to undergo reversible structural changes in response to changes in temperature or chemical environment. ${ }^{14}$ These structural 
changes have been described as "breathing modes", in which the lattice distortions lead to significant changes in pore size. As an example, the 1-D diamond-shaped pores of MIL53, a chromium-carboxylate MOF, undergoes significant and reversible dimensional changes (over $5 \AA$ ) during the hydration/dehydration process (Figure 7). This breathing process could potentially be exploited to selectively trap and release analytes in MOF frameworks. Finally, luminescent phosphors can be integrated into the MOF framework as a route to develop sorbents that can be sensors for particular analytes. ${ }^{15}$ Recent work has demonstrated that lanthanide SBUs can be design so retain their luminescent properties within a crystalline lattice structure. Since the electronic transitions of these

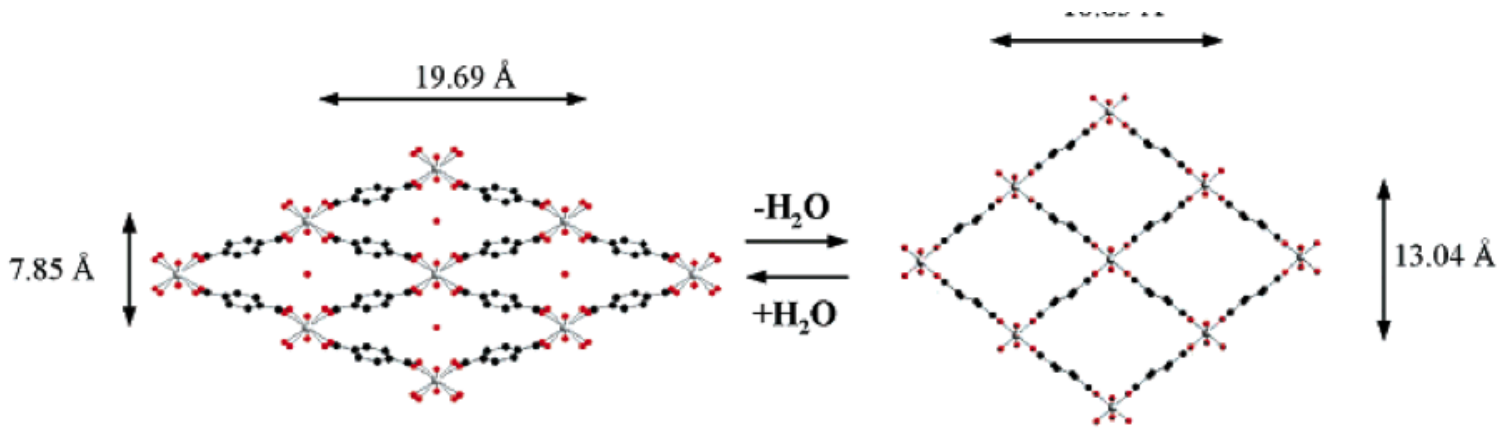

Figure 7. Graphical representation of the "breathing" effect that occurs in MIL-53 during the reversible hydration/dehydration process (ref. 14).

metals are sensitive to their local environment, changes in their emission spectra can be used to monitor binding of guest molecules to the MOF structure.

Acknowledgement. This work was performed under the auspices of the U.S. Department of Energy by Lawrence Livermore National Laboratory under Contract DE-AC52-07NA27344 and funded by the DOE Office of Energy Efficiency and Renewable Energy. 


\section{References}

1. For some recent reviews, see: (a) Kuppler, R. J.; Timmons, D. J.; Fang, Q.; Li, J.; Makal, T. A.; Young, M. A.; Yuan, D.; Zhao, D.; Zhuang, W.; Zhou, H. Coord. Chem. Rev. 2009, 253, 3042. (b) Ferey, G. Chem. Soc. Rev. 2008, 37, 191. (c) Roswell, J. L. C.; Yaghi, O. M. Micro. Mesopor. Mater. 2004, 73, 3.

2. (a) Long, J. R.; Yaghi, O. M. Chem. Soc. Rev. 2009, 38, 1213. (b) Ockwig, N. W.; Delgado-Friedrichs, O.; O'Keefe, M.; Yaghi, O. M. Acc. Chem. Res. 2005, 38, 176.

3. (a) Roswell, J. L. C.; Millward, A. R.; Park, K. S.; Yaghi, O. M. J. Am. Chem. Soc. 2004, 126, 5666. (b) Kaye, S. S.; Dailly, A.; Yaghi, O. M.; Long, J. R. J. Am. Chem. Soc. 2007, 129, 14176.

4. Yaghi, O. M.; Li, Q. MRS Bulletin, 2009, 34, 682.

5. Chae, H. K.; Siberio-Perez, D. Y.; Kim, J.; Go, Y.; Eddaoudi, M.; Matzger, A. J.; O’Keefe, M.; Yaghi, O. M. Nature 2004, 427, 523.

6. Morris, W.; Doonan, C. J.; Furukawa, H.; Banerjee, R.; Yaghi, O. M. J. Am. Chem. Soc, 2008, 130, 12626.

7. (a) Horike, S.; Dinca, M.; Tamaki, K.; Long, J. R. J. Am. Chem. Soc. 2008, 130, 5854. (b) Vimont, A.; Goupil, J.; Lavalley, J.; Daturi, M. Surbie, S.; Serre, C.; Millange, F.; Ferey, G.; Audebrand, N. J. Am. Chem. Soc. 2006, 128, 3218.

8. Ferey, G.; Mellot-Draznieks, C.; Serre, C.; Millange, F.; Dutour, J.; Surble, S.; Margiolaki, I. Science, 2005, 309, 2040.

9. Chui, S. S.; Lo, S. M.; Charmant, J. P. H.; Orpen, A. G.; Williams, I. D. Science, 1999, 283, 1148.

10. Rosi, N. L. Kim, J.; Eddaoudi, M.; Chen, B.; O’Keeffe, M.; Yaghi, O. M. J. Am. Chem. Soc. 2005, 127, 1504.

11. Britt, D.; Tranchemontagne, D.; Yaghi, O. M. Proc. Nat. Acad. Sci. 2008, 105, 11623.

12. (a) Wang, B.; Cote, A. P.; Furukawa, H.; O'Keefe, M.; Yaghi, O. M. Nature, 2008, 453, 207. (b) Park, K. S.; Ni, Z.; Cote, A. P.; Choi, J. Y.; Huang, R.; UribeRomo, F. J.; Chae, H. K.; O'Keefe, M.; Yaghi, O. M. Proc. Nat. Acad. Sci. 2006, $103,10186$. 
13. (a) El-Kaderi, H. M.; Hunt, J. R.; Mendoza-Cortes, J. L.; Cote, A. P.; Taylor, R. E.; O’Keefe, M.; Yaghi, O. M. Science, 2007, 316, 268. (b) Cote, A. P.; Benin, A. I.; Ockwig, N. W.; O’Keefe, M.; Matzger, A. J.; Yaghi, O. M. Science, 2005, 310, 1166. (c) Han, S. S.; Furukawa, H.; Yaghi, O. M.; Goddard, W. A. J. Am. Chem. Soc. 2008, 130, 11580.

14. Serre, C.; Millange, F.; Thouvenot, C.; Nogues, M.; Marsolier, G.; Louer, D.; Ferey, G. J. Am. Chem. Soc. 2002, 124, 13519.

15. Chandler, B. D.; Yu, J. O.; Cramb, D. T.; Shimizu, G. K. H. Chem. Mater. 2007, $19,4467$. 